\title{
THE DYNAMICAL EVOLUTION OF CENTAURUS A
}

\author{
S.G. VINE AND R.C. THOMSON \\ Institute of Astronomy \\ Madingley Road, Cambridge, CB3 OHA. UK.
}

\section{Centaurus A and NGC 5237 - A Spiral-Elliptical Interaction}

Centaurus A (NGC 5128) is a nearby Giant Elliptical (3.5Mpc, $10^{12}$ Solar Masses). It has a prominent dust-lane which has an axis of rotation orientated differently from that of the underlying galaxy. Close to it are two galaxies: NGC 5237 - a dwarf elliptical, and Fourcade-Figueroa - a low surface brightness irregular. We are investigating whether these three objects are the resulting components of a grazing interaction between a Giant Elliptical and a less massive spiral galaxy. We have conducted n-body simulations, modelling all components of the two original interacting galaxies. Preliminary results show that Cen A, its dust-lane, and NGC 5237 are consistent with this simulation model. Centaurus A exhibits many other features which are also indicative of a past interaction.

Thomson (1992, MNRAS, 257, 689) conducted test-particle simulations to investigate the Spiral-Elliptical interaction. Here we report much more sophisticated simulations which mimic both the elliptical and the spiral by a gravitationally self-consistent model. In our model the elliptical is ten times as massive as the spiral galaxy. The components of the spiral: bulge, disc, and dark matter halo are populated by particles according to the observed density and velocity distributions. The spiral and elliptical have initially a parabolic path, with the spiral in a prograde sense grazing the maximum radius of the elliptical.

We have used the HARP n-body integrator. This is a piece of hardware which was developed to calculate the integration algorithm of Makino and Aarseth $(1992$, PASJ, 44, 141). It can be thought of as a "hardware subroutine" called from simulation code. It's speed enables us to explore a wide range of initial parameters which would otherwise be a severe restriction in this approach to the problem. 